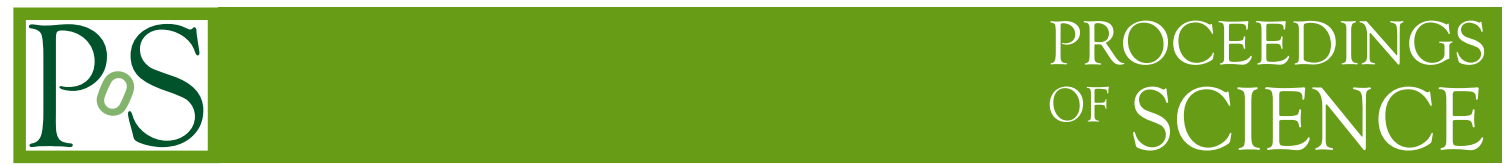

\title{
Searches for leptoquarks in CMS
}

\author{
Francesco Romeo* on behalf of the CMS Collaboration \\ Vanderbilt University \\ E-mail: fromeodcern.ch
}

\begin{abstract}
The most recent results from searches for leptoquarks are presented using $p p$ collision data collected by the CMS experiment at a centre-of-mass energy of $13 \mathrm{TeV}$. A variety of final states is considered, probing leptoquark couplings to a quark and a neutrino, a top quark and a muon, a $\mathrm{b}$ quark and a tau lepton, and a light-flavour quark and a muon or an electron. No evidence is observed for physics beyond the standard model and 95\% confidence level (C.L.) limits are set on model parameters for scalar and vector leptoquarks.
\end{abstract}

European Physical Society Conference on High Energy Physics - EPS-HEP2019 -

10-17 July, 2019

Ghent, Belgium

${ }^{*}$ Speaker. 


\section{Introduction}

The "leptoquarks" (LQ) are hypothetical particles that carry both lepton and baryon number. They are predicted in many extensions of the standard model (SM) of which grand unified theories (GUT) [1], composite models [2], extended technicolor models [3], and R parity violating supersymmetry (SUSY) [4] are some examples.

LQ have fractional electric charge, and can have spin 0 (scalar LQ, denoted LQ ${ }_{S}$ ) or 1 (vector $\mathrm{LQ}$, denoted $\mathrm{LQ}_{V}$ ). At hadron colliders, they can be produced mainly in a pair or singly, in association with a lepton, as illustrated in the Feynman diagrams in Fig. 1. For pair produced LQs, the cross section depends only on the LQ mass for LQs, while for LQv it may depend from additional parameters, in order to comply with constraints imposed by unitarity at high energy scales. In the following, we will consider the dimensionless coupling $k$ and the value 1, Yang-Mills case, and 0 , minimal coupling case. For singly produced LQ, the cross section further depends on the coupling to quark and lepton, $\lambda$, and the quark generation.
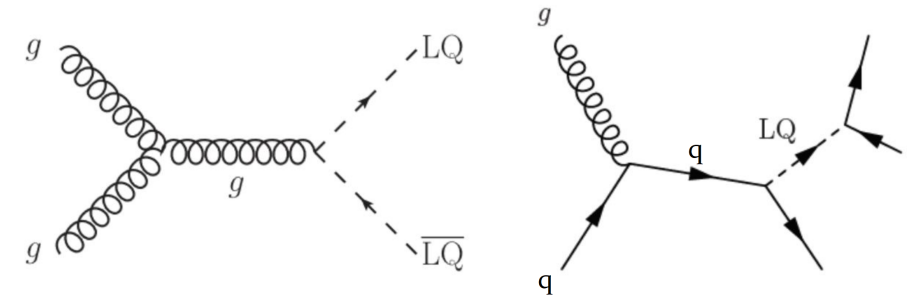

Figure 1: Feynman diagram for the production of pair (left) and single (right) LQ.

The LQ have recently gained enhanced interest as they may provide an explanation to a series of anomalies observed in precision measurements in the B-physics sector [5].

In this proceeding, we describe the most recent results from searches of LQ using $p p$ collision data collected by the CMS experiment [6] at a centre-of-mass energy of $13 \mathrm{TeV}$. A vast range of signatures is considered in order to probe several LQ couplings, including those to a quark and a neutrino, a top quark and a muon, a b quark and a tau lepton, and a light-flavour quark and a muon or an electron. A complete description of the objects used in the analyses described below as well as of the systematic uncertainties treatment can be found in the corresponding references.

\section{Search for LQ coupling to the pair $q v$}

In this section the search for LQ coupling to the pair $q v$ is described, whose complete description can be found in [7]. The analysis reinterprets the results of a generic search for squarks and gluinos [8] to set constraints on pair produced $\mathrm{LQ}_{S}$ and $\mathrm{LQ}_{V}$.

The baseline selection requires $N_{j} \geq 1$, where $N_{j}$ denotes the number of jets with transverse momentum (pT) $>30 \mathrm{GeV}$, and to pass either missing transverse energy $\left(p_{T}^{\text {miss }}\right)>30 \mathrm{GeV}$ if they have $\mathrm{HT}>1000 \mathrm{GeV}$, or $p_{T}^{\text {miss }}>250 \mathrm{GeV}$ if they have $250<H T<1000 \mathrm{GeV}$, being HT the scalar sum of jet pT. Further baseline requirements include that the $p_{T}^{\text {miss }}$ vector is not aligned in the azimuthal angle $\phi$ with any of the four leading jets in pT, that the negative vector sum of jet transverse momenta is consistent with the $p_{T}^{m i s s}$, and that no loosely identified charged leptons or isolated tracks are found in the event. Events are then categorized according to four variables: HT, $M_{T 2}$ [8], $N_{j}$, and number of b-tagged jets. The analysis spans a wide range of kinematics and jet 

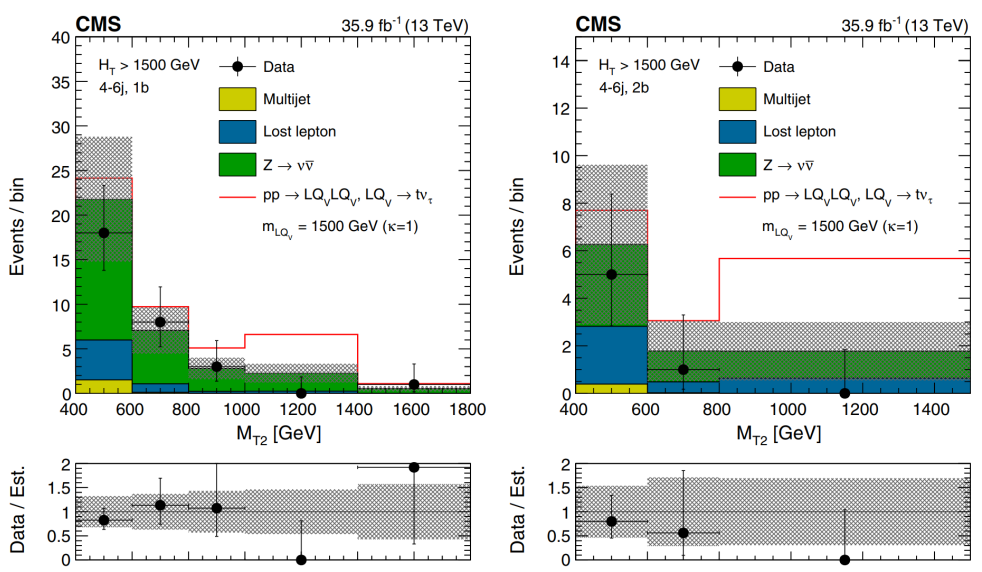

Figure 2: Distributions of $\mathrm{M}_{T 2}$ showing data (black points), the background predictions (stack plot), and a hypothetical $\mathrm{LQ}_{V}$ signal with LQ mass of $1500 \mathrm{GeV}$ for two categories with $\mathrm{H}_{T}>1500 \mathrm{GeV}, 4 \leq \mathrm{N}_{j} \leq 6$, and $\mathrm{N}_{b}=1$ (left) and $\mathrm{N}_{b}=2$ (right).
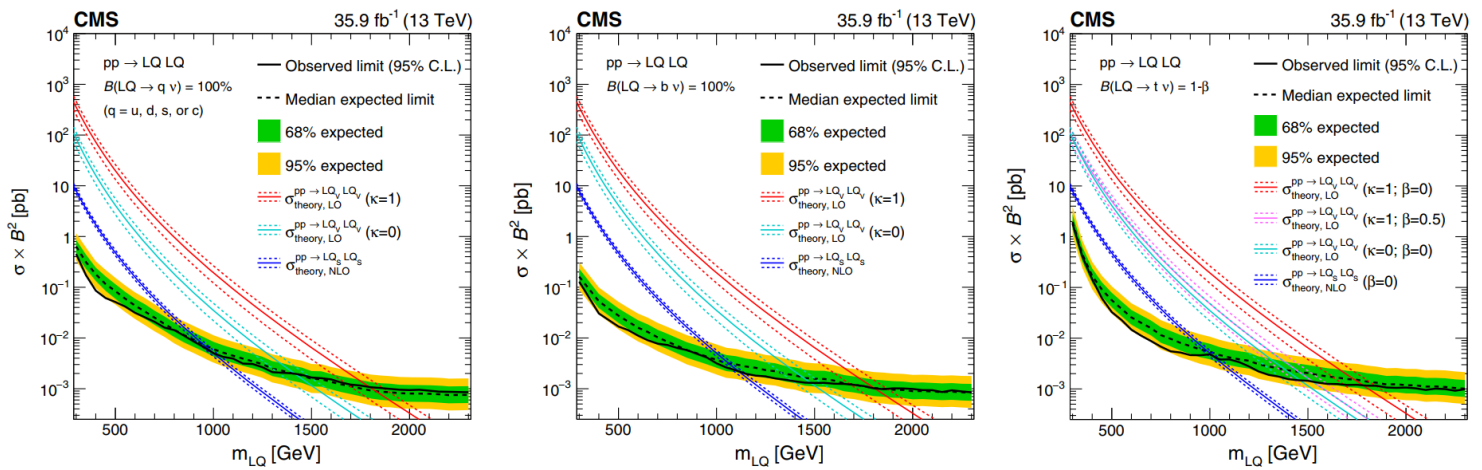

Figure 3: The 95\% C.L. upper limits on the production cross sections as a function of LQ mass for LQ pair production decaying with $100 \%$ branching fraction to a neutrino and (left) a light quark (one of u, d, s, or c), (center) a bottom quark, or (right) a top quark.

multiplicities, containing 213 search bins in total. Figure 2 shows the $M_{T 2}$ distribution in two of the most sensitive search categories for the mass of the LQ $\left(\mathrm{m}_{L Q}\right)=1500 \mathrm{GeV}$ and the LQ decaying with unity branching fraction to the pair $t v$. Assuming a scalar (vector) LQ decaying with unity branching fraction to a light quark and neutrino, $\mathrm{m}_{L Q}<980$ (1790) $\mathrm{GeV}$ are excluded at the $95 \%$ C.L. by the observed data. For an LQ coupling to $b v, \mathrm{~m}_{L Q}<1100$ (1810) $\mathrm{GeV}$ are excluded, and for an LQ decaying to $t v, \mathrm{~m}_{L Q}<1020$ (1780) $\mathrm{GeV}$ are excluded, as shown in Figure 3.

\section{Search for LQ coupling to the pair $t \mu$}

Here, we report the results on the search for LQ coupling to the pair $t \mu$, whose complete description is in [9].

The analysis considers pair produced LQ in the final state with $2 t$ plus $2 \mu$. Events are selected considering $e$ and $\mu$ with $p_{\mathrm{T}}>30 \mathrm{GeV}$ and if they have a minimum $\mathrm{S}_{T}$, the scalar $p_{\mathrm{T}}$ sum of all selected final state objects, of $350 \mathrm{GeV}$ and dimuon mass of $110 \mathrm{GeV}$. They are then separated in 2 categories according on whether they have at least 3 leptons or not. 
The signal extraction is performed relying on the distribution of the LQ mass $\mathrm{M}_{L Q}^{r e c}$ (3 lepton category) and $S_{T}$ that are shown in Fig. 4. From Figure 4 a good agreement between the data and the expected background is found. Results are interpreted reporting observed upper limits on the production cross section for pair produced $\mathrm{LQ}_{S}$ and $\mathrm{LQ}_{V}$ at $95 \%$ C.L. in the plane $\mathrm{B}(\mathrm{LQ} \rightarrow t \mu)$ $\mathrm{M}_{L Q}$ that includes results from a search for LQ coupling to the pair $t \tau$ [10], Figure 5 (left), and $\mathrm{B}(\mathrm{LQ} \rightarrow t \mu)-\mathrm{M}_{L Q}$ that includes results from a search for LQ coupling to the pair $b v$ [7], Figure 5 (right).
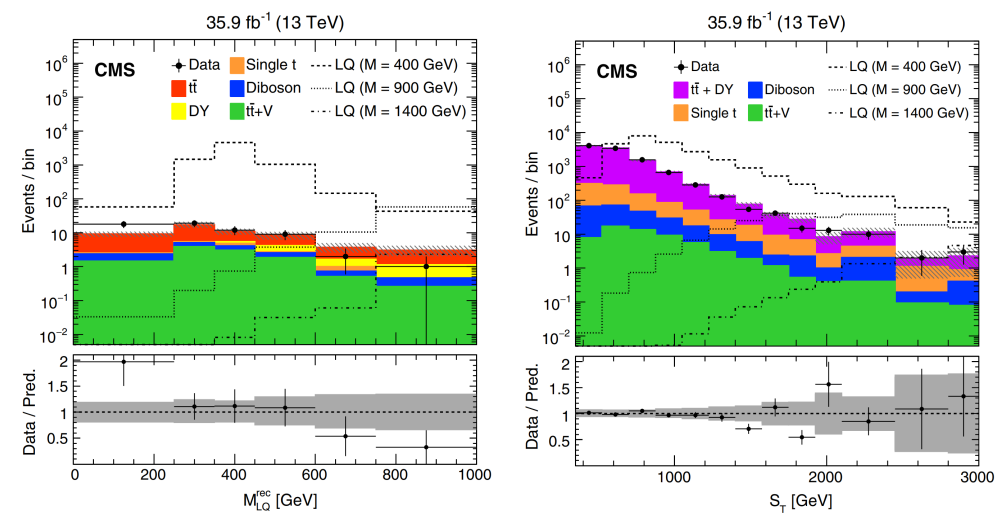

Figure 4: Distributions of the leptoquark mass, $\mathrm{M}_{L Q}^{r e c}$, (left) and the $\mathrm{S}_{T}$ variable (right), defined in the text.
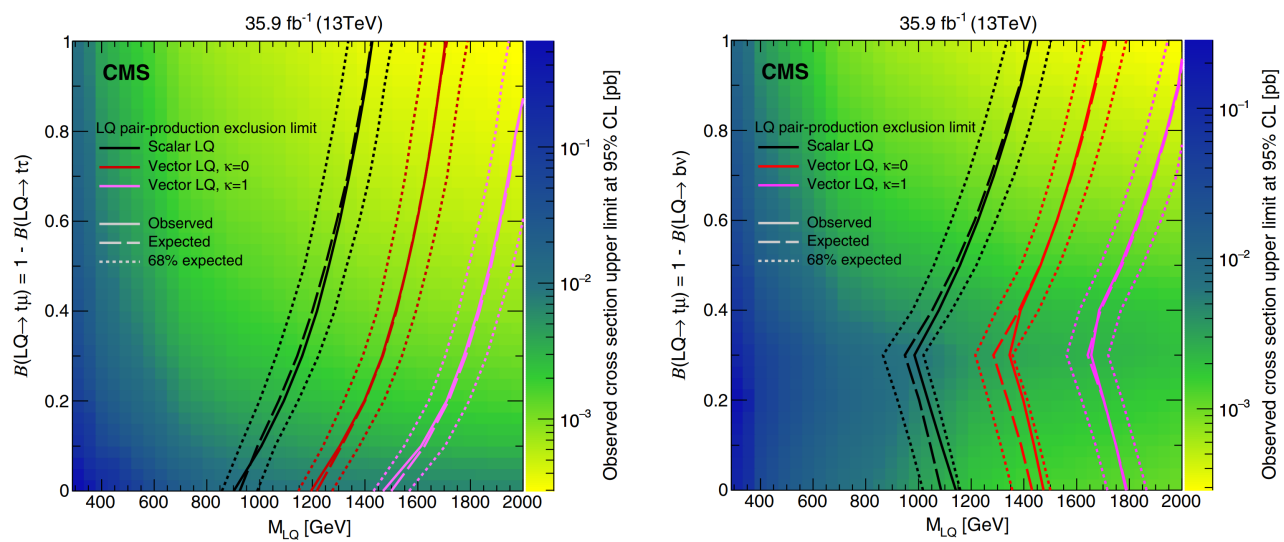

Figure 5: Observed upper limits on the production cross section for pair production of LQ decaying into a top quark and a muon or a $\tau$ lepton (left) and LQ decaying into a top quark and a muon or into a bottom quark and a neutrino (right) at $95 \%$ C.L. in the $M_{L Q}-\mathscr{B}(L Q \rightarrow t \mu)$ plane.

\section{Search for LQ coupling to the pair $b \tau$}

The case of an LQ coupling to the pair $b \tau$ is investigated through the pair production and the single production mechanism, whose cross-section competes with that of pair produced LQ for some values of $\lambda$ and $\mathrm{m}_{L Q}$ of $\mathrm{O}(1) \mathrm{TeV}$ [11]. The analyses consider the final states with $2 \tau$ leptons plus $2 \mathrm{~b}$ quarks and $2 \tau$ leptons plus $1 \mathrm{~b}$ quarks, whose complete description is reported in [12] and [13].

An excess of events over the SM backgrounds is searched for using the distribution of $\mathrm{S}_{T}$ and $\mathrm{S}_{T}^{M E T}$, which is given by $\mathrm{S}_{T}$ plus $p_{T}^{m i s s}$. 
Figure 6 shows the $S_{T}$ distribution of data and SM prediction for the pair (left) and singly (right) produced LQ searches, with the observed data consistent with the background only (SM) hypothesis. Figure 7 illustrates the 95\% C.L. upper limits up to masses of $1 \mathrm{TeV}$ for pair produced $\mathrm{LQ}_{S}$ (left) and in the plane $\lambda-\mathrm{m}_{L Q}$ (right) for singly produced $\mathrm{LQ}_{S}$.
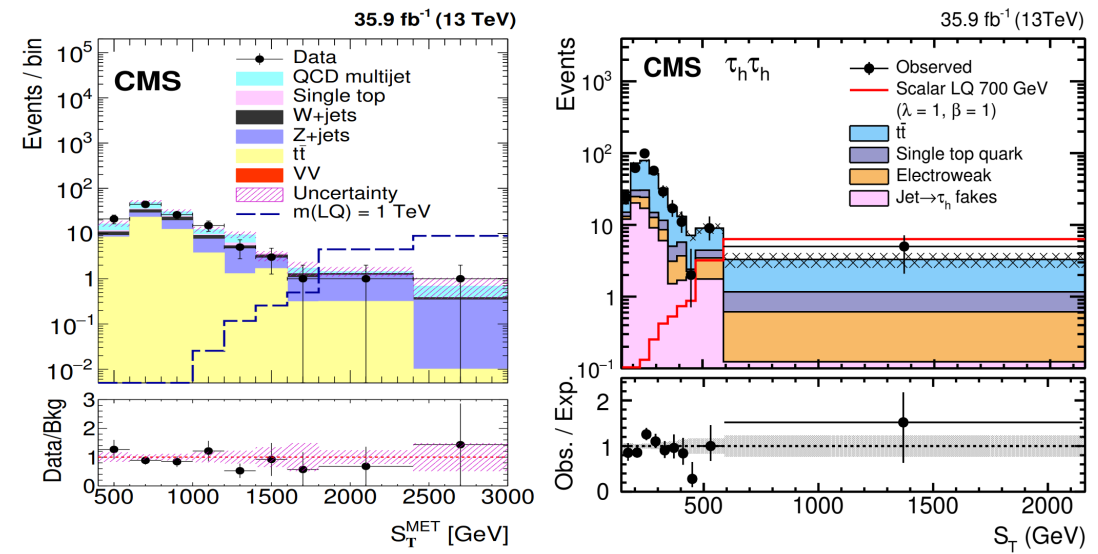

Figure 6: Distributions of $S_{T}$ for the search of pair produced LQ (left) and singly produced LQ (right).
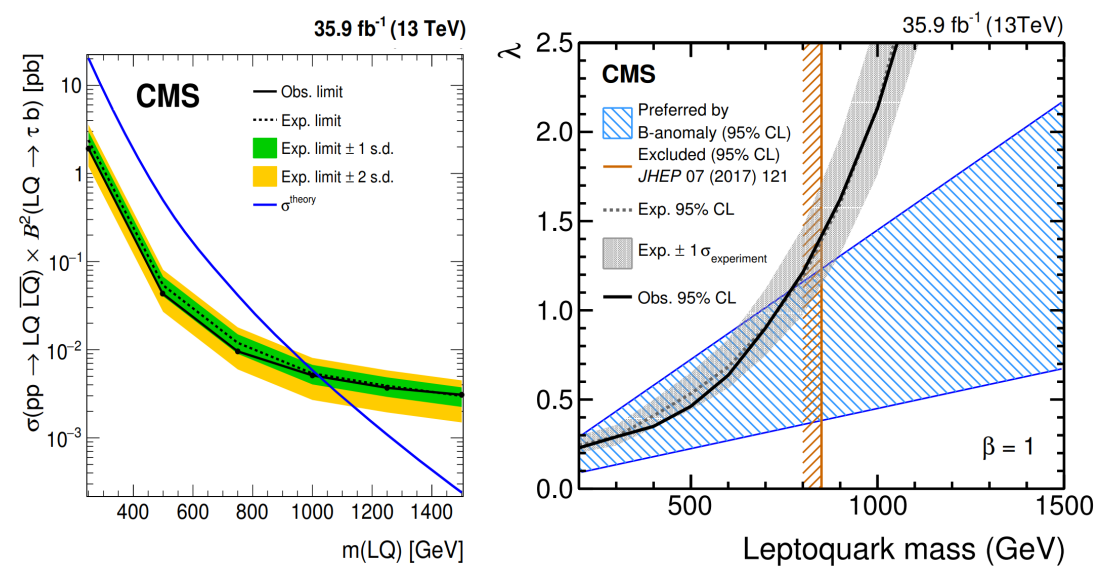

Figure 7: The 95\% C.L. upper limits (left) on the production cross sections as a function of LQ mass for LQ $s$ pair production (left) and on the Yukawa coupling $\lambda$ at the LQ-lepton-quark vertex, as a function of the LQ mass, from the search of singly produced $\mathrm{LQ}_{S}$ (right).

\section{Search for LQ coupling to the pair $q \ell(q v)$}

The LQ coupling to the pair $q \ell(q v)$ is looked for considering the final states with $2 e$ plus 2 jets ( $1 e$ plus $p_{T}^{\text {miss }}$ plus 2 jets) and $2 \mu$ plus 2 jets ( $1 \mu$ plus $p_{T}^{\text {miss }}$ plus 2 jets), whose complete searches are described in [14] and [15].

Electrons, muons, and jets are selected requiring their $p_{\mathrm{T}}$ to be greater than $50 \mathrm{GeV}$, which is the same minimum value required for $p_{T}^{\text {miss }}$ in the event. The final signal selection is optimized for the highest significance considering 3 event variables: the mass of the 2 leptons (the transverse mass of the lepton plus $\left.p_{T}^{m i s s}\right), \mathrm{S}_{T}$, and $\mathrm{m}_{\ell j}^{\min }$, which is obtained from the smallest jet- $\ell$ pair that minimizes the LQ mass difference of the possible pairs. 
Figure 8 shows the distrubtion of $\mathrm{m}_{\ell j}^{\min }$ for the electron (left) and muon (right) channels, with a good agreement between the data and the SM prediction. Figure 9 reports the $95 \%$ C.L. for pair produced LQ $\mathrm{L}_{S}$ for the $2 e$ plus 2 jets and $1 e$ plus $p_{T}^{\text {miss }}$ plus 2 jets (left) and $2 \mu$ plus 2 jets and $1 \mu$ plus $p_{T}^{\text {miss }}$ plus 2 jets (right) shown in the plane of the branching fraction $\beta=\mathscr{B}(\mathrm{LQ} \rightarrow \ell q)(1-\beta=$ $\mathrm{B}(\mathrm{LQ} \rightarrow v q))$ versus the mass of the LQ.
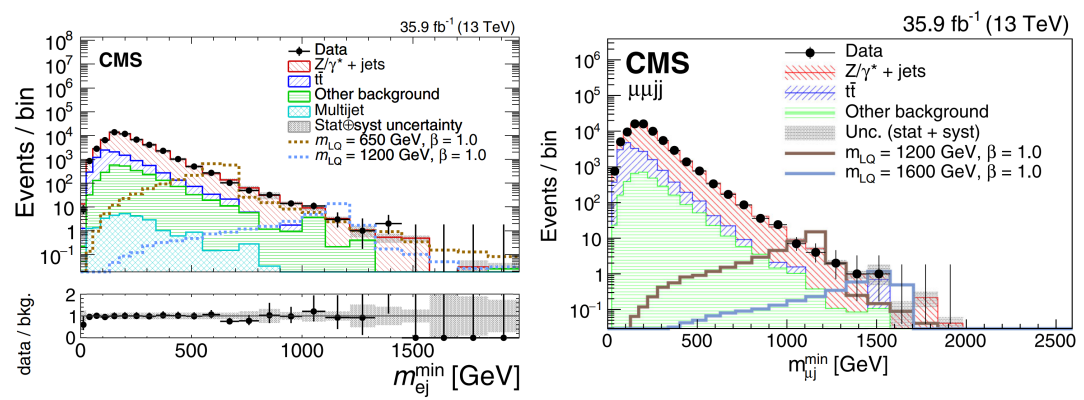

Figure 8: Distributions of $\mathrm{m}_{\ell j}^{\min }$ for the case of $\ell=e$ (left), $2 e$ plus 2 jets search, and $\ell=\mu$ (right), $2 \mu$ plus 2 jets search.
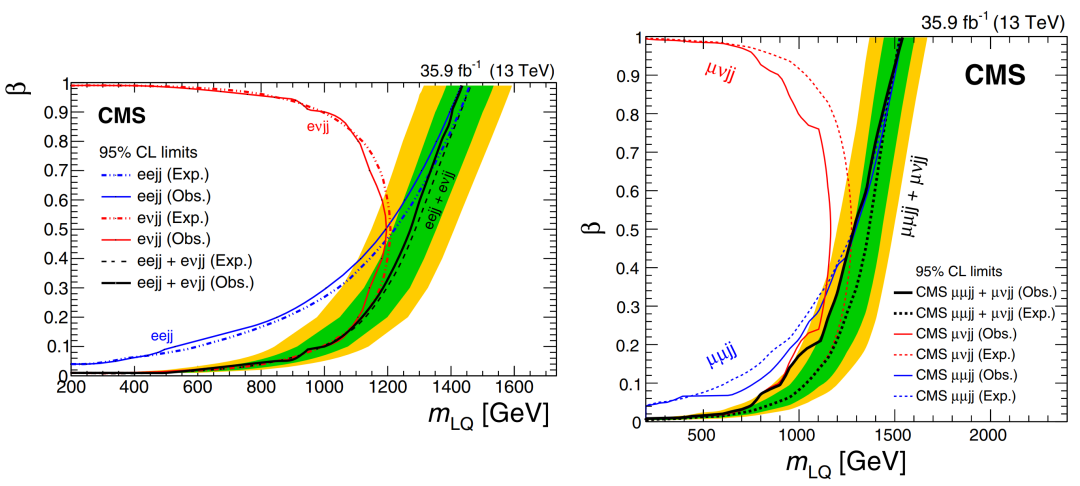

Figure 9: The 95\% C.L. for pair produced scalar LQ coupling to $e q(v q)$ (left) and $\mu q(v q)$ (right) shown in the plane of the branching fraction $\beta=\mathscr{B}(\mathrm{LQ} \rightarrow \ell q)(1-\beta=\mathrm{B}(\mathrm{LQ} \rightarrow v q))$ versus the mass of the $\mathrm{LQ}$.

\section{Summary}

In this proceeding we have reported the state of the art in the CMS experiment of searches for LQ, investigating different lepton plus jet signatures. In all the searches a good agreement is found between the data and the expectation from the SM. The diagram in Fig. 10 summarizes the 95\% C.L. exclusion limits on the mass of the LQ, either scalar or vector, for the coupling to different lepton-quark pairs and the corresponding final states investigated.

\section{References}

[1] J. C. Pati and A. Salam, "Unified lepton-hadron symmetry and a gauge theory of the basic interactions," Phys. Rev. D, vol. 8, p. 1240, 1973.

[2] B. Schrempp and F. Schrempp, “Light leptoquarks,” Phys. Lett. B, vol. 153, p. 101, 1985.

[3] S. Dimopoulos and L. Susskind, "Mass without scalars," Nucl. Phys. B, vol. 155, p. 237, 1979. 


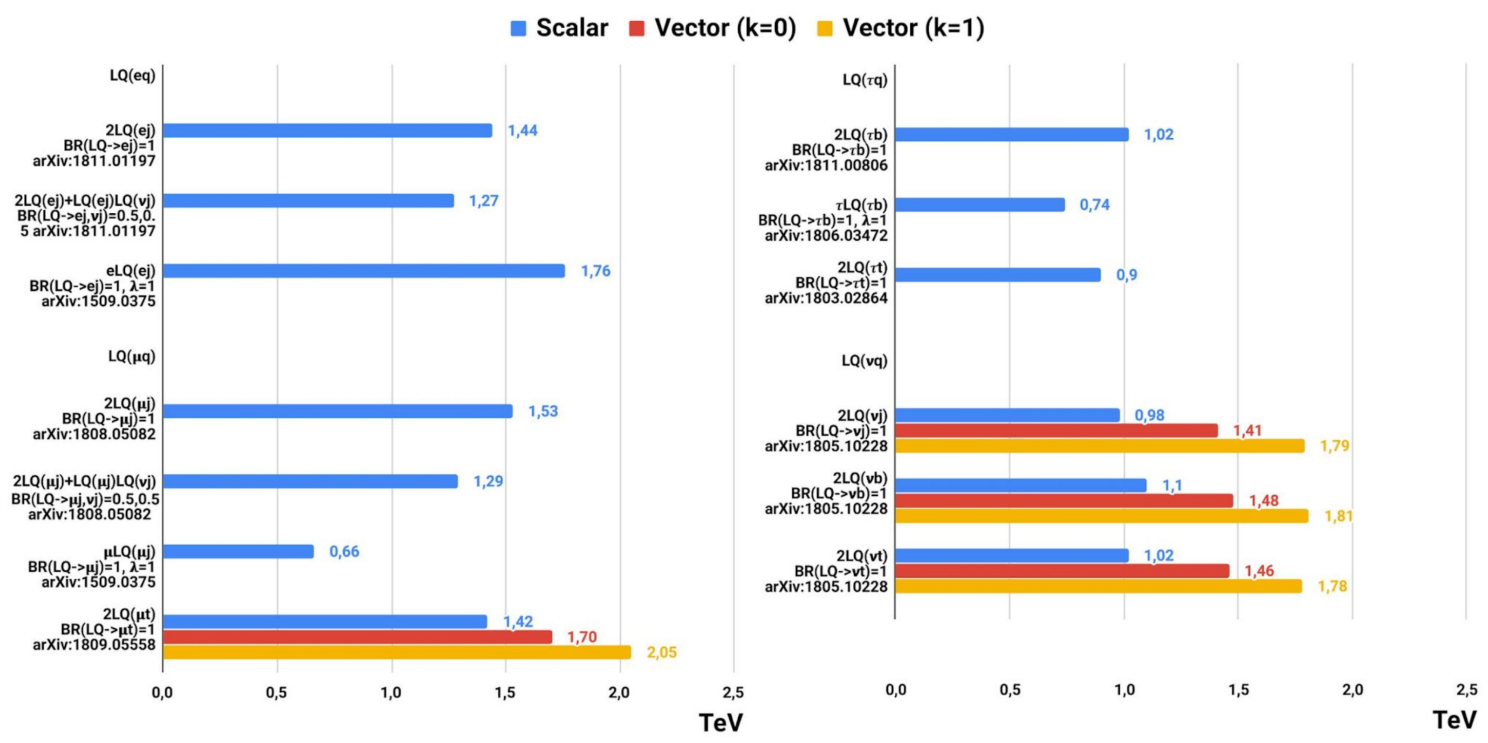

Figure 10: Summary of the exclusion limits on the LQ mass for different hypotheses of the coupling to a quark-lepton pair and the nature of the LQ, scalar or vector $(\mathrm{k}=1$, Yang-Mills case, and 0 , minimal coupling case).

[4] G. R. Farrar and P. Fayet, "Phenomenology of the production, decay, and detection of new hadronic states associated with supersymmetry," Phys. Lett. B, vol. 76, p. 575, 1978.

[5] J. Aebischer, W. Altmannshofer, D. Guadagnoli, M. Reboud, P. Stangl, and D. M. Straub, “ $B$-decay discrepancies after Moriond 2019," 2019.

[6] CMS Collaboration, "The CMS experiment at the CERN LHC," JINST, vol. 3, p. S0800, 2008.

[7] CMS Collaboration, "Constraints on models of scalar and vector leptoquarks decaying to a quark and a neutrino at $\sqrt{s}=13 \mathrm{TeV}$," Phys. Rev., vol. D98, no. 3, p. 032005, 2018.

[8] CMS Collaboration, "Search for new phenomena with the $M_{\mathrm{T} 2}$ variable in the all-hadronic final state produced in proton-proton collisions at $\sqrt{s}=13 \mathrm{TeV}$," Eur. Phys. J., vol. C77, no. 10, p. 710, 2017.

[9] CMS Collaboration, "Search for leptoquarks coupled to third-generation quarks in proton-proton collisions at $\sqrt{s}=13$ TeV," Phys. Rev. Lett., vol. 121, no. 24, p. 241802, 2018.

[10] CMS Collaboration, "Search for third-generation scalar leptoquarks decaying to a top quark and a $\tau$ lepton at $\sqrt{s}=13 \mathrm{TeV}$," Eur. Phys. J., vol. C78, p. 707, 2018.

[11] D. Buttazzo, A. Greljo, G. Isidori, and D. Marzocca, "B-physics anomalies: a guide to combined explanations," JHEP, vol. 11, p. 044, 2017.

[12] CMS Collaboration, "Search for a singly produced third-generation scalar leptoquark decaying to a $\tau$ lepton and a bottom quark in proton-proton collisions at $\sqrt{s}=13 \mathrm{TeV}$, , JHEP, vol. 07, p. 115, 2018.

[13] CMS Collaboration, "Search for heavy neutrinos and third-generation leptoquarks in hadronic states of two $\tau$ leptons and two jets in proton-proton collisions at $\sqrt{s}=13 \mathrm{TeV}$," JHEP, vol. 03, p. 170, 2019.

[14] CMS Collaboration, "Search for pair production of first-generation scalar leptoquarks at $\sqrt{s}=13$ TeV," Phys. Rev., vol. D99, no. 5, p. 052002, 2019.

[15] CMS Collaboration, "Search for pair production of second-generation leptoquarks at $\sqrt{s}=13 \mathrm{TeV}$," Phys. Rev., vol. D99, no. 3, p. 032014, 2019. 